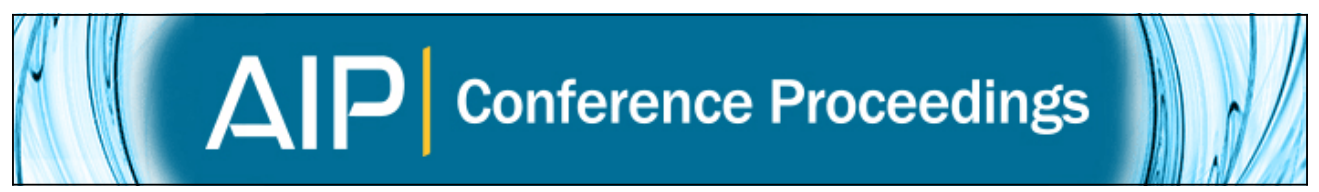

Implementation of Absorbing Boundary Conditions for the Einstein Equations

Oliver Rinne, Luisa T. Buchman, Mark A. Scheel, and Harald P. Pfeiffer

Citation: AIP Conference Proceedings 1122, 384 (2009); doi: 10.1063/1.3141332

View online: http://dx.doi.org/10.1063/1.3141332

View Table of Contents:

http://scitation.aip.org/content/aip/proceeding/aipcp/1122?ver=pdfcov

Published by the AIP Publishing

Articles you may be interested in

Will Gravitational Waves Confirm Einstein's General Relativity?

AIP Conf. Proc. 1168, 1090 (2009); 10.1063/1.3241244

Apparent horizons in D-dimensional Robinson-Trautman spacetime

AIP Conf. Proc. 1122, 404 (2009); 10.1063/1.3141339

The Causal Boundary Of Standard Stationary Spacetimes

AIP Conf. Proc. 1122, 272 (2009); 10.1063/1.3141290

Constructing Traversable Wormholes: An Initial Value Approach

AIP Conf. Proc. 1122, 260 (2009); 10.1063/1.3141284

Pseudo-Hermiticity versus PT symmetry: The necessary condition for the reality of the spectrum of a non-Hermitian Hamiltonian

J. Math. Phys. 43, 205 (2002); 10.1063/1.1418246 


\title{
Implementation of Absorbing Boundary Conditions for the Einstein Equations
}

\author{
Oliver Rinne*,†, Luisa T. Buchman**,\$, Mark A. Scheel** and Harald \\ P. Pfeiffer** \\ ${ }^{*}$ Department of Applied Mathematics and Theoretical Physics, Centre for Mathematical Sciences, \\ Wilberforce Road, Cambridge CB3 OWA, UK \\ ${ }^{\dagger}$ King's College, Cambridge CB2 IST, UK \\ ${ }^{* *}$ Theoretical Astrophysics 130-33, California Institute of Technology, Pasadena, CA 91125, USA \\ "Center for Relativity, University of Texas at Austin, Austin, TX 78712, USA
}

\begin{abstract}
Based on a recent study of the linearized Bianchi equations by Buchman and Sarbach, we construct and implement a hierarchy of absorbing boundary conditions for the Einstein equations in generalized harmonic gauge. As a test problem, we demonstrate that we can evolve multipolar gravitational waves without any spurious reflections at linear order in perturbation theory.
\end{abstract}

Keywords: Numerical relativity, initial-boundary value problem, perturbation theory

PACS: 04.25.D-, 02.60.Lj, 04.25.-g

\section{INTRODUCTION}

In numerical evolutions of asymptotically flat spacetimes describing isolated selfgravitating systems, one is faced with the problem of how to treat an unbounded spatial domain with finite computer resources. The standard approach is to adopt the Cauchy formulation of general relativity and to truncate the spatial domain at a finite distance, where boundary conditions (BCs) must be imposed. These BCs must (i) be constraint preserving, (ii) yield a well-posed initial-boundary value problem, and (iii) minimize spurious reflections of gravitational radiation, i.e. the BCs must be absorbing.

Based on a study of the linearized Bianchi equations, Buchman and Sarbach [1, 2] constructed a hierarchy of absorbing BCs $\mathscr{B}_{L}$ that are perfectly absorbing for all radiation with angular momentum numbers $\ell \leqslant L$, where $L$ is an arbitrary given number. The objective of the present work is to incorporate these BCs into a complete set of constraint-preserving BCs for the Einstein equations, and to implement and test these $\mathrm{BCs}$ numerically. This requires a suitable reformulation of the BCs; our algorithm is outlined in Sec. 2.

As a first test problem for our numerical implementation, we consider multipolar (angular momentum number $\ell$ ) linearized gravitational waves in transverse-traceless gauge $[3,4]$. We demonstrate that with our absorbing BC $\mathscr{B}_{L=\ell}$, we can evolve these without any spurious reflections to linear order in perturbation the ory (Sec. 3). In contrast, the BCs $\mathscr{B}_{L}$ with $L<\ell$ are not compatible with the exact linearized solution. This includes the often-used $B C$ that freezes the Newman-Penrose scalar $\Psi_{0}$ at the boundary, which corresponds precisely to $\mathscr{B}_{1}$.

The use of absorbing $\mathrm{BCs}$ is crucial if one wants to extract radiation in a reliable 
way close to the outer boundary, e.g. for gravitational wave data analysis. The most sophisticated $\mathrm{BC}$ currently in use for binary black hole simulations is $\mathscr{B}_{1}$. For a given wavelength and outer boundary location, the reflection coefficient of this $\mathrm{BC}$ increases with $\ell$ [1]; hence our higher-order absorbing $\mathrm{BCs}$ will be particularly important when there is significant higher-multipolar radiation. This is the case for unequal mass binaries: e.g. for a mass ratio of $M_{1} / M_{2}=4$, the fraction of the energy emitted in modes with $\ell>2$ exceeds $10 \%[5]$.

For further details on our work, the reader is referred to Ref. [6].

\section{FORMULATION OF THE BOUNDARY CONDITIONS}

We assume that close to the outer boundary, spacetime may be linearized about Minkowski space, and that the outer boundary is spherical. (In future work we plan to include first-order corrections due to a Schwarzschild background [2].) While the original Buchman-Sarbach BCs $\mathscr{B}_{L}$ were formulated in terms of the Newman-Penrose scalar $\Psi_{0}$, we choose to work with the closely related Regge-Wheeler-Zerilli (RWZ) scalars $\Phi_{\ell m}^{( \pm)}$[7]. The indices $\ell m$ refer to an expansion in spherical harmonics and $( \pm)$ refer to the parity; we suppress these indices in the following. The RWZ scalars have the advantage of obeying a closed wavelike evolution equation,

$$
\left[\partial_{t}^{2}-\partial_{r}^{2}+\frac{\ell(\ell+1)}{r^{2}}\right] \Phi=0 .
$$

The BCs $\mathscr{B}_{L}$ turn out to be equivalent to the well-known Bayliss-Turkel [8] BCs for the scalar wave equation (see e.g. Ref. [9] for a numerical implementation),

$$
\mathscr{B}_{L}: \quad\left[r^{2}\left(\partial_{t}+\partial_{r}\right)\right]^{L+1} \Phi \hat{=} 0,
$$

where $\hat{=}$ denotes equality on the boundary. The higher-order radial derivatives make these $\mathrm{BCs}$ difficult to implement numerically. To eliminate these derivatives, we introduce a set of auxiliary variables at the boundary [10],

$$
w_{k} \equiv r^{-(2 k+1)}\left[r^{2}\left(\partial_{t}+\partial_{r}\right)\right]^{k} \Phi .
$$

These can be shown to obey the following system of ODEs intrinsic to the boundary,

$$
\left(\partial_{t}+\frac{k}{r}\right) w_{k}=\frac{1}{2 r^{2}}[-\ell(\ell+1)+k(k-1)] w_{k-1}+\frac{1}{2} w_{k+1} .
$$

The absorbing $\mathrm{BC} \mathscr{B}_{L}$ is equivalent to setting $w_{L+1} \hat{=} 0$, and together with $w_{0}=\Phi / r$ this closes the system of ODEs (4).

Our algorithm now consists of three basic steps:

1. Extraction of the RWZ scalars from the spacetime metric,

2. Evolution of the auxiliary system (4) on the boundary,

3. Construction of boundary data for the Einstein equations from the auxiliary system. 
Details of each of these steps can be found in Ref. [6]. We use a first-order symmetric hyperbolic formulation of the Einstein equations in generalized harmonic gauge. The BCs we impose on these equations are of the form of those proposed in Ref. [11] but with a special choice of boundary data. We split the incoming characteristic fields into three categories: (i) constrained fields, on which constraint-preserving $\mathrm{BCs}$ are imposed, (ii) physical fields, for which we construct boundary data from the auxiliary system at the boundary that implements the desired absorbing BC, and (iv) gauge fields, which reflect the remaining gauge freedom in generalized harmonic gauge. We have checked that our results presented below in Sec. 3 are insensitive to the particular choice of gauge BC.

\section{NUMERICAL TESTS}

We have implemented the algorithm outlined above using a pseudo-spectral collocation method, the Caltech-Cornell Spectral Einstein Code (SpEC). The numerical domain is decomposed into a central sphere and a series of spherical shells. The outer boundary is placed at a radius $R=30$.

As a test problem, we consider linearized multipolar gravitational waves in transversetraceless gauge. These were first derived explicitly for $\ell=2$ by Teukolsky [3] and have recently been generalized to arbitrary $\ell>2$ in Ref. [4]. We choose a purely outgoing wave with radial profile

$$
F_{\text {out }}(r-t)=A \exp \left[-\frac{\left(r-t-r_{0}\right)^{2}}{\sigma^{2}}\right],
$$

where $r_{0}=15$ and $\sigma=1.5$. We specify initial data for the Einstein equations corresponding to this exact linearized solution and then evolve them numerically.

In order to measure the spurious reflections caused by the outer boundary, we numerically extract the RWZ scalars at the boundary and compare them with the exact linearized solution. Since the exact solution is only valid in linearized theory whereas we solve the full nonlinear Einstein equations, the two will necessarily disagree because of nonlinear effects. In order to separate these from the effects at linear order in perturbation theory in which we are interested, we run the simulations with various values of the amplitude $A$ in Eq. (5). The results for an odd-parity wave with $\ell=m=2$ are shown in Fig. 1. The difference between the numerical and exact RWZ scalar plotted here has been normalized by the amplitude, so that if the quantity plotted still decreases at least linearly with amplitude, the numerical and exact solution agree at linear order in perturbation theory. This is the behavior seen in the left panel of Fig. 1, which uses $\mathscr{B}_{2}$. This result confirms the theoretical prediction that $\mathscr{B}_{2}$ is perfectly absorbing for this solution. In contrast, for $\mathscr{B}_{1}$ (right panel) we find that the curves begin to overlap as the amplitude is decreased, indicating a discrepancy between the exact and numerical solution at linear order in perturbation theory, in accordance with the prediction.

Similar results for higher multipoles $(\ell=3,4)$ are included in Ref. [6]. We have also computed a numerical approximation to the reflection coefficients of the various BCs and find good agreement with the analytical results of Ref. [1] in the region where the numerical solution is not contaminated by nonlinear effects or roundoff error. 

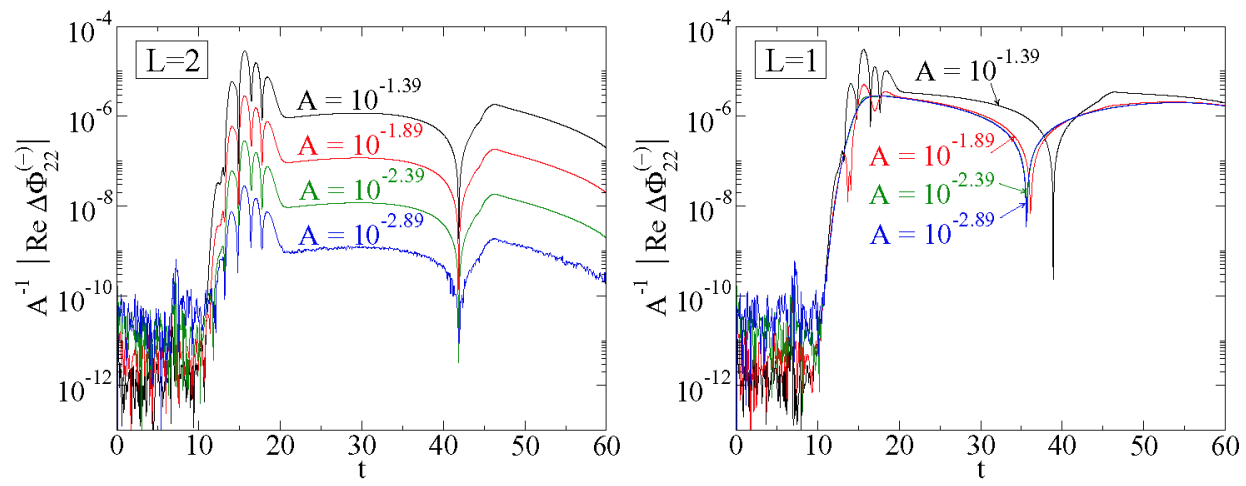

FIGURE 1. Difference between the numerically-extracted RWZ scalar $\Phi_{22}^{(-)}$and its exact linearized value, evaluated on the outer boundary, and divided by the amplitude $A$. The exact linearized solution and the initial data for the evolution contain only the $\ell=2, m=2$ odd-parity mode. The different panels correspond to different orders $L$ of the absorbing $\mathrm{BC} \mathscr{B}_{L}, L=2$ (left) and $L=1$ (right).

\section{ACKNOWLEDGMENTS}

We thank James Bardeen, Edvin Deadman, Lee Lindblom, Richard Matzner, Olivier Sarbach, John Stewart, and Manuel Tiglio for insightful suggestions and discussions during the course of this work, and Keith Matthews for use of and help with his ODE integration code. The numerical simulations presented here were performed using the Spectral Einstein Code (SpEC) developed at Caltech and Cornell primarily by Larry Kidder, Harald Pfeiffer and Mark Scheel.

OR gratefully acknowledges a travel grant from the Gravitational Waves Section of the European Network of Theoretical Astroparticle Physics / ILIAS to attend this conference, as well as a Research Fellowship at King's College, Cambridge. This work was supported in part by grants to Caltech from the Sherman Fairchild Foundation and the Brinson Foundation, by NSF grants DMS-0553302, PHY-0601459, PHY-0652995, and by NASA grant NNG05GG52G. LTB was also supported by grants NSF PHY 03 54842 and NASA NNG 04GL37G to the University of Texas at Austin.

\section{REFERENCES}

1. L. T. Buchman and O. C. A. Sarbach, Class. Quantum Grav. 23, 6709-6744 (2006).

2. L. T. Buchman and O. C. A. Sarbach, Class. Quantum Grav. 24, S307-S326 (2007).

3. S. A. Teukolsky, Phys. Rev. D 26, 745-750 (1982).

4. O. Rinne (2008), arxiv: 0809.1761.

5. E. Berti et al., Phys. Rev. D 76, 064034 (2007).

6. O. Rinne, L. T. Buchman, M. A. Scheel, and H. P. Pfeiffer (2008), arxiv: 0811.3593.

7. O. Sarbach and M. Tiglio, Phys. Rev. D 64, 084016 (2001).

8. A. Bayliss and E. Turkel, Comm. Pure Appl. Math. 33, 707-725 (1980).

9. J. Novak and S. Bonazzola, J. Comput. Phys. 197, 86-196 (2004).

10. T. Hagstrom and S. Hariharan, Appl. Numer. Math. 27, 403-416 (1998).

11. H.-O. Kreiss and J. Winicour, Class. Quantum Grav. 16, S405-S420 (2006). 